\title{
Hypertransaminasemia in poorly-controlled type-1 diabetes mellitus
}

\author{
C. Merino Palacios, J. Primo Vera ${ }^{1}$ J. Fernández Chinchilla ${ }^{1}$, J. Ferrando Marco², M. Aragó Galindo and \\ L. García Ferrer
}

Service of Internal Medicine, ${ }^{1}$ Division of Gastroenterology and ${ }^{2}$ Department of Pathology. Hospital de Sagunto. Valencia. Spain

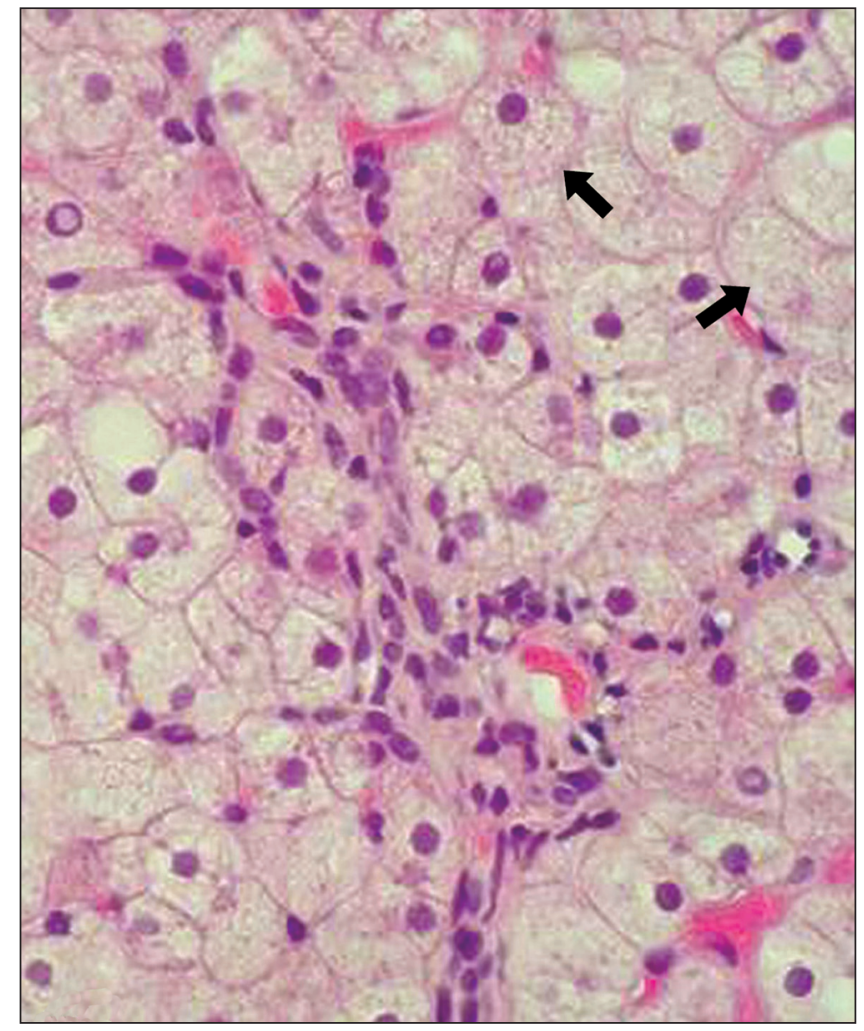

Fig. 1.-

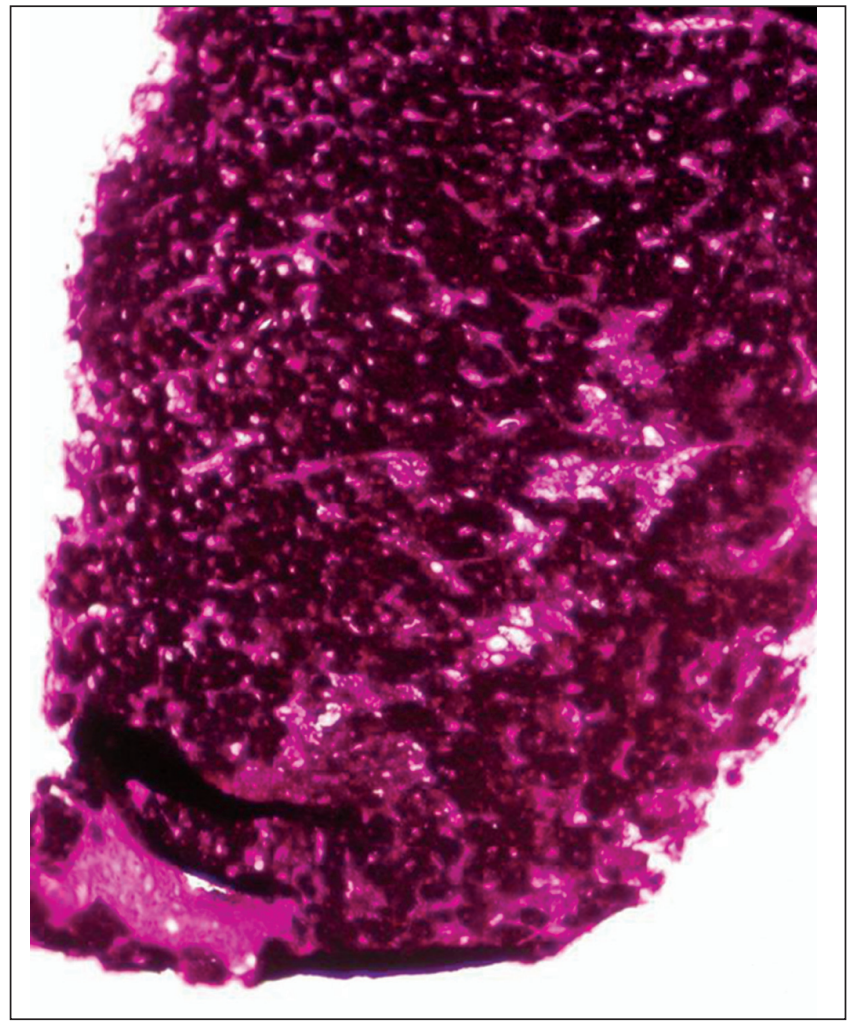

Fig. 2.-

A 16-year-old boy had been diagnosed with type-1 diabetes mellitus at the age of 9 with no subsequent appropriate metabolic control (HbA1c: 10-11\%). He presented frequent hypoglycemic episodes and required hospitalization in several occasions because of vomiting and ketoacidosis. His weight and height were in low percentiles (BMI: 21.1 $\mathrm{kg} / \mathrm{m}^{2}$ ) for his age and laboratory data did not disclosed any abnormality except those related with the diabetes. While remaining asymptomatic, an ordinary laboratory test revealed abnormal liver function parameters (AST/ALT: 578/526 U/1, GGT: $284 \mathrm{U} / \mathrm{l}$ ) and a raised lipid profile (cholesterol/triglycerides 225/223: mg/dl). An initial study yielded negative results (virus serologic tests, autoantibodies, electrophoresis, complement, alpha1 antitrypsin, and celiac disease screening tests). For the following 8 months, the patients remained asymptomatic, although with a bad control of the diabetes and elevated serum levels of aminotransferases (AST 59-227 IU/1, ALT 68-120 IU/1 y GGT 53-120 IU/l). At that moment, physical examination revealed the presence of a hepatomegaly and a new investigation demonstrated positive ANA (1/640), but other autoantibodies were (SMA, LKM-1, AMA) negative. His HLA haplotype was DR4. Abdominal ultrasonography demonstrated a diffuse hyperechogenic liver suggestive of fatty infiltration. A liver biopsy revealed a normal liver architecture with mild inflammatory infiltrates in some portal tracts and 
no piecemeal necrosis. Liver cells showed a pale and microvacuolated cytoplasm (arrow in Fig. 1, hematoxylin and eosin x 200) without additional hepatic injury. These cytoplasmic deposits were PAS-positive (Fig. 2) and not visible following diastase digestion indicating the presence of deposits of liver glycogen and excluding hepatic steatosis. In conclusion, this patient with poor controlled type-1 diabetes mellitus developed a hepatic glycogenosis resembling Mauriac's syndrome (1). This complication can revert following optimal control of the glycemia (2).

\title{
REFERENCES
}

1. Chatila R, West AB. Hepatomegaly and abnormal liver test due to glycogenosis in adults with diabetes. Medicine (Baltimore) 1996; 75: 327-33.

2. Munns CF, McCrossin RB, Thomsett MJ, Batch J. Hepatic glycogenosis: reversible hetomegaly in type 1 diabetes. J Paediatr Child Health 2000; 36: 44952.

\section{Hipertransaminasemia y diabetes mellitus no controlada}

\author{
C. Merino Palacios, J. Primo Vera ${ }^{1}$ J. Fernández Chinchilla ${ }^{1}$, J. Ferrando Marco², M. Aragó Galindo y \\ L. García Ferrer
}

Servicios de Medicina Interna, ${ }^{1}$ Unidad de Digestivo $y^{2}$ Anatomía Patológica. Hospital de Sagunto. Valencia

Varón de 16 años diagnosticado de diabetes mellitus tipo 1 desde los 9 años, con un control metabólico inadecuado (HbA1c 10-11\%). Presentaba frecuentes episodios de hipoglucemias e ingresos hospitalarios por vómitos y en una ocasión por cetoacidosis diabética. En dichos ingresos, la exploración física tan sólo mostraba unos percentiles bajos de estatura y peso para su edad (IMC $21,1 \mathrm{~kg} / \mathrm{m}^{2}$ ) y en la analítica no destacaba ninguna alteración salvo las relacionadas con su diabetes.

En un control rutinario, estando asintomático, se detectó una alteración de la función hepática y del perfil lipídico (AST $578 \mathrm{IU} / \mathrm{l}$, ALT $526 \mathrm{IU} / \mathrm{l}$, GGT $284 \mathrm{IU} / \mathrm{l}$, colesterol $225 \mathrm{mg} / \mathrm{dl}$, triglicéridos $223 \mathrm{mg} / \mathrm{dl}$ ). El estudio inicial para confirmar la presencia de hepatopatía y buscar su etiología fue negativo (marcadores serológicos víricos, autoanticuerpos, proteinograma, complemento, alfa-1-antitripsina, cribado de enfermedad celiaca). Durante los 8 meses siguientes el paciente continuó asintomático, con un mal control metabólico y persistiendo la hipertrasaminasemia (AST 59-227 U/l, ALT 68-120 U/1 y GGT 53-120 U/1). En ese momento destacaba en la exploración física una discreta hepatomegalia. Se realizó un nuevo estudio que incluyó los parámetros anteriores y la determinación de inmunoglobulinas, ceruloplasmina, cobre y metabolismo férrico, resultando normales a excepción de los anticuerpos antinucleares (ANA 1/640), siendo el resto de autoanticuerpos (SMA, LKM-1, AMA) negativos. Era portador del haplotipo DR4. La ecografía hepática mostró un hígado ligeramente aumentado e hiperecogénico, sugestivo de infiltración grasa. Se realizó biopsia hepática que reveló una arquitectura conservada, con mínimo infiltrado inflamatorio en algún espacio porta sin afectar a la limitante. Los hepatocitos presentaron un aspecto claro y microvacuolado (Fig. 1 tinción hematoxilina-eosina), comprobándose la existencia de abundantes depósitos de material PAS positivo en el citoplasma (Fig. 2), que no eran visibles tras la digestión con diastasa. Ello confirmaba la presencia de glucógeno y descartaba esteatosis. Se trata por tanto de una glucogenosis hepática adquirida en un paciente diabético con mal control metabólico compatible con síndrome de Mauriac (1). Este trastorno es reversible con un control metabólico estricto (2). 